\title{
Cognitive flexibility improves memory for delayed intentions
}

Seth R. Koslov ${ }^{1}$, Arjun Mukerji' ${ }^{2}$, Katlyn R. Hedgpeth ${ }^{1}$, and Jarrod A. Lewis-Peacock ${ }^{1}$

${ }^{1}$ Department of Psychology, Institute for Neuroscience, University of Texas, Austin, Texas, ${ }^{2}$ Helen Wills Neuroscience Institute, University of California (UC), Berkeley, California

\author{
Corresponding Author: \\ Seth R. Koslov \\ Department of Psychology \\ University of Texas at Austin \\ Austin, TX 78712 \\ Tel: +1512 232-1805 \\ koslov@utexas.edu
}




\section{Abstract}

Cognitive control involves the allocation of cognitive resources in order to successfully navigate and interact with the world. Oftentimes, control involves balancing the demands brought on by performing immediately relevant tasks and those required in order to perform future intended actions. For example, directing attention towards navigating through traffic on a highway while also needing to remember to exit the freeway at a particular street. This ability to delay execution of a goal until the appropriate time in the future is referred to as prospective memory (PM). The dual mechanisms of cognitive control (DMC) framework posits that individuals can use two different strategies to remember an intended action: a proactive control strategy involving working memory maintenance of the goal and monitoring of the environment, or a reactive control strategy relying on timely retrieval of goal information from episodic memory. Previous research on prospective memory has demonstrated that performance improves when individuals engage these control strategies in accordance with the demands of the task environment. However, it is unclear how people select a control strategy, particularly in situations with dynamic task demands. We hypothesized that if people flexibly adapt their strategy in response to changes in the environment, this should facilitate prospective memory. Across two experiments, we asked participants to identify the reappearance of a picture target (a prospective memory intention) while at the same time performing an ongoing visual search task. The attentional demands of the ongoing task were manipulated to monotonically increase or decrease on a moment to moment basis. The selection of control strategies was identified using reaction time costs and neural measures of intention maintenance. 
Results showed that people fluidly modified control strategies, shifting towards proactive control when the attentional demands decreased, and shifting towards reactive control when attentional demands increased. Critically, these adaptive shifts in control strategy were associated with better prospective memory performance. These results demonstrate that fine-grained control of attention and memory resources serves an adaptive role for remembering to carry out future plans. 


\section{Introduction}

Life is busy and keeping track of what we are doing and what we intend to do can

3 be challenging. Cognitive control describes the set of processes by which we are able to

4 maintain and connect goals to actions and to subsequently filter out irrelevant

5 distractors in accordance with these goals ${ }^{1}$. Juggling goals in spite of interruptions is a

6 ubiquitous part of everyday life ${ }^{2}$, constituting upwards of $50-80 \%$ of our daily memory

7 problems ${ }^{3,4}$. The cognitive ability to delay the execution of a goal until a precise moment

8 is known as prospective memory (PM). For example, remembering to pick up groceries

9 on your way home from work later today requires PM. How do you accomplish this? The

10 multiprocess theory of PM, which is a specific PM-focused implementation of the more

11 general dual methods of cognitive control framework (DMC $\left.{ }^{5-7}\right)$, describes two

12 dissociable strategies: proactive control and reactive contro/ ${ }^{8,9}$. Proactive control relies

13 on working memory to remember the goal and external attention to monitor the

14 environment for cues to act ${ }^{9-12}$. Reactive control relies on episodic memory to store the

15 goal and salient cues from the environment to trigger its timely retrieval ${ }^{13-15}$.

16 These strategies have been shown to have distinct behavioral and neural

17 profiles. Proactive control is cognitively demanding ${ }^{6,16}$ and interferes with ongoing

18 processing ${ }^{10,17}$, whereas reactive control relies less on working memory processing and

19 can succeed without any observable interference costs ${ }^{18-23}$. Further evidence for this

20 dissociation comes, in part, from observations of individual and age-related differences

21 in PM strategy capabilities. Individual differences in working memory have been more

22 strongly linked to proactive than reactive performance ${ }^{24-27}$. Additionally, age-related

23 impairments in PM, rooted in declining cognitive control capabilities, have been linked to 
24 proactive control deficits ${ }^{28-33}$, but not to deficits in reactive control ${ }^{34,35}$. Dissociable

25 neural correlates have also been identified for these strategies ${ }^{36-40}$. In summary,

26 proactive PM performance is primarily related to sustained activity in anterior prefrontal

27 cortex, as well as other regions of the frontoparietal control network ${ }^{41}$, and to working

28 memory maintenance in inferior temporal regions ${ }^{37}$. The engagement of reactive control

29 mechanisms in response to PM events have been linked to increased connectivity to ${ }^{39}$

30 and transient responses in the middle temporal gyrus ${ }^{38}$, as well as transient responses

31 in the precuneus, ventral parietal cortex, and precuneus ${ }^{36,41}$.

32 The DMC posits that successful cognition depends on optimizing the use of

33 proactive and reactive control in response to environmental demands. Preparatory

34 processes involved in proactive control, like strategic monitoring of the environment for

35 intention-relevant cues, may benefit PM, but also place high costs on working memory

36 and attentional capacities. In low-demand environments, controlled attentional

37 processes can be successfully allocated to maintain goal information in working

38 memory and to strategically monitor the environment for the right time and place to

39 act $^{6,42,43}$. Therefore, in situations of low working memory load or a high probability of a

40 PM event, proactive control should be beneficial. However, when working memory load

41 is high, it is more efficient to offload the PM intention to the reactive control system and

42 redirect cognitive resources towards more immediate demands ${ }^{5}$. For example, when

43 concurrent cognitive demands are high - as one might experience as traffic congestion

44 increases on the commute home - offloading an important PM intention to reactive

45 control (e.g., remembering to stop at the grocery store to buy orange juice) frees

46 resources for other urgent demands (safely navigating the heavy traffic). While reactive 
47 control is less cognitively demanding, it is more susceptible to proactive interference

48 and more vulnerable to lapses in attention to goal-relevant events in the environment ${ }^{6,44}$

49 (e.g., failing to notice the turnoff for the grocery store and proceeding home sans orange

50 juice). It is therefore important to select a control strategy best suited to the current

51 situation in order to reduce the risk that prospective intentions interfere with more urgent

52 demands, and also to reduce the risk that those intentions go unfulfilled.

53 One major area of research in PM has been to explain how individuals choose

54 and change strategies in response to variable environmental demands ${ }^{45}$. Guynn ${ }^{11,46}$

55 proposed that PM performance is in part supported by two dissociable components of

56 proactive control: an all-or-nothing sustained prospective intention component and a

57 more flexible prospective monitoring component. The prospective intention component

58 is described as load-invariant and relative inflexible, while the monitoring component is

59 thought to be dynamically sensitive to environmental factors ${ }^{47}$. Recent related work has

60 suggested that the sustained intention component of this model is also able to be

61 strategically adjusted to some extent ${ }^{16,48}$, and that the amount of cognitive resources

62 needed to maintain the intention-state can negatively affect monitoring ability ${ }^{49}$. This

63 monitoring plus intention-state theory of PM doesn't explicitly account for reactive

64 control, but it acknowledges that there are certainly times when spontaneous retrieval

65 (i.e. reactive control) may be accounting for PM performance.

Many models of PM have focused on the relationship between proactive and

67 reactive control. Some models propose a central executive process that allocates

68 resources either towards proactive or reactive control along a continuum ${ }^{50}$, or strikes

69 some balance between attention to external stimuli (ongoing demands) or internal 
70 stimuli (PM intention maintenance $)^{41}$. A recent update of dual-process PM theory, the

71 dynamic multiprocess framework (DMPV ${ }^{51,52}$ ), proposes that people have a flexible

72 choice between proactive control and reactive control that primarily depends on the

73 contextual likelihood of a PM event. According to this model, individuals will rely on

74 reactive control when the probability of a PM event is low, but then temporarily "switch

75 on" proactive control when a cue in the environment signals an increased likelihood of

76 the PM event (in support of this theory see ${ }^{53-55}$ ). While DMPV hypothesizes a fluid

77 relationship between proactive and reactive control modes, this relationship has yet to

78 be demonstrated. To date, empirical work has only shown that people can perform a

79 bimodal switch between the two strategies following abrupt changes in the environment.

80 In real-world settings, however, the task environment (e.g., the ongoing demands on our

81 cognitive resources) may not always change so abruptly, but rather may fluctuate more

82 gradually from moment to moment. It is unclear how people adjust their PM strategy

83 when situational demands change dynamically over time. For example, remembering to

84 exit the highway at the correct street for the grocery store on the way home while

85 dealing with the ebbs and flows of traffic congestion.

86 Across two experiments, we evaluated how individuals adjusted their PM control

87 strategies when cognitive demands increased or decreased over time and in turn how

88 this impacted prospective remembering. We hypothesized that adapting one's control

89 strategy to better align with the cognitive load caused by competing demands (e.g.,

90 using a more proactive strategy when attentional resources become available to do so,

91 and using a more reactive strategy when fewer attentional resources are available)

92 should improve prospective remembering. We modified our existing PM paradigm 
93 (Lewis-Peacock et al., $2016^{37}$ ) and required participants to make a delayed target

94 detection with pictures of faces and scenes while also performing an ongoing visual-

95 search task with oriented arrows. The paradigm was modified so that the cognitive

96 demands within a trial could be parametrically varied to create some situations that

97 favor a more proactive control strategy for remembering the target picture (decreasing

98 ongoing demands), and other situations that favor a more reactive control strategy

99 (increasing ongoing demands). The cognitive demands of this task were manipulated by

100 subtly, but monotonically, adjusting task difficulty within each trial. For information on

101 the pilot studies used to validate the task design, see supplementary materials.

We collected two measures of PM strategy use on every trial - a behavioral

103 index (RTs on the ongoing task) and a neural metric (fMRI pattern classification of the

104 PM intention). Our previous work ${ }^{37}$ suggests that these two measures of PM strategy

105 provide unique and complementary assessments of memory performance in situations

106 with stable cognitive demands, and we anticipated they would also be informative in

107 situations with less stable demands. The RT measure is a behavioral hallmark of

108 proactive control as it reflects dual-task interference costs ("PM costs") arising from the

109 active maintenance and monitoring of a PM intention ${ }^{8,10,56}$. The neural measure, derived

110 from multivariate classifier analysis of fMRI data, has been associated with the working

111 memory maintenance of PM intentions ${ }^{37,57-59}$. In a first experiment, we linked time-

112 sensitive behavioral measures of changes in PM strategy within a trial to memory

113 performance $(n=50)$. We then replicated this experiment while also collecting fMRI

114 data $(n=28)$. This allowed us to combine neural measures of PM intention processing

115 with behavioral measures of shifts in PM strategy. Performance was similar across both 
116 Experiments 1 and 2, therefore all behavioral data were pooled for subsequent analyses

117 (see supplementary materials for separated analyses of behavioral and fMRI samples).

118 To evaluate the consequences of cognitive flexibility in dynamic environments, we

119 linked these behavioral and neural measures of PM strategy to PM accuracy on a trial120 by-trial basis.

\section{Results}

\section{Task Overview}

124 The dual-task paradigm used here to evaluate PM strategy selection and 125 modification was adapted from the design of Lewis-Peacock et al. (2016) $)^{37}$. Participants 126 solved a delayed target recognition task for a picture of a face or a scene (the PM task) 127 while concurrently performing a visual search task every $2 \mathrm{~s}$ on an array of tilted arrows 128 (the ongoing task; Figure 1, see supplementary materials for more details on task 129 design). The ongoing task required participants to indicate whether a rightward facing 130 horizontal arrow was present or absent in a circular array of arrows. Ongoing task (OG) 131 difficulty was manipulated by adjusting the degree to which the angles of distractors 132 were similar to the horizontal plane. Difficulty could increase or decrease across a trial, 133 or remain fixed at the middle difficulty level (difficulty level 8 out of 15). For increasing 134 and decreasing trials, difficulty shifted monotonically at the rate of one difficulty level 135 every $2 \mathrm{~s}$ (i.e. shifted on each new probe display). Trials began with the presentation of 136 the trial-unique PM target, followed by 1 to 15 probe displays. On each probe display, 137 participants could respond either "present" or "absent" to the ongoing task, or "target" to 138 the PM task, but not both. PM targets appeared on the last probe of each trial, and were 139 followed by PM-task feedback (2s), and then by a break (6s) before the next trial. 

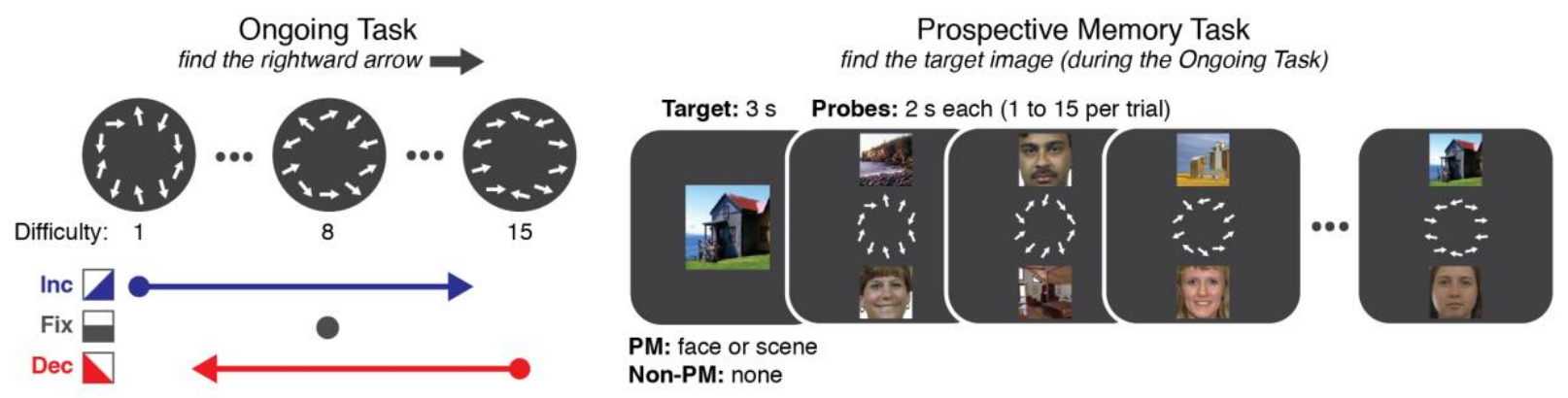

Figure 1. Task design. Left: Ongoing task difficulty could increase or decrease every $2 \mathrm{~s}$ across a trial or remain fixed at the middle difficulty level (8 of 15). Right: In the dualtask PM experiment, participants identified the reappearance of a PM target while concurrently performing the ongoing task.

Participants performed well on the ongoing $(O G)$ task (mean accuracy $=83.64 \%$, $95 \% \mathrm{Cl}=[75.49 \%, 91.79 \%])$ as summarized in Figure 2. There was no interaction between trial type (PM trials versus non-PM trials) and task difficulty $\left(\beta\right.$ interaction $=6.2^{*} 10^{-}$ ${ }^{4}, 95 \% \mathrm{Cl}=[-0.001,0.002]$, pinteraction $\left.=0.468\right)$ on OG task accuracy. OG accuracy

151 decreased as difficulty increased, and there was a small, but reliable main effect of trial type between $\mathrm{PM}$ and non-PM trials $\left(\beta\right.$ diff $=-.026,95 \% \mathrm{Cl}=[-0.027,-0.025], p_{\text {diff }}<.001$;

$153 \beta_{\mathrm{pm}}=0.008,95 \% \mathrm{Cl}=[0.001,0.016], \mathrm{p}_{\mathrm{pm}}=0.024 ;$ marginal $\mathrm{r}^{2}=0.57 ;$ Figure $\left.1 \mathrm{~A}\right)$. The

154 main effect of trial type indicated a dip in OG accuracy of less than $1 \%$ on PM trials

155 compared to non-PM trial. A follow-up analysis compared OG accuracies from PM and non-PM trials at each difficulty level, finding that accuracies were only reliably different

157 at difficulty level 8 on fixed difficulty trials $(p=0.025$, after Bonferroni correction factor

158 15). On dynamic trials (increasing and decreasing difficulty), the main effect of PM task

159 on OG accuracy was not significant $\left(\beta_{\mathrm{pm}}=0.007,95 \% \mathrm{Cl}=\left[-8.5^{*} 10^{-5}, 0.015\right], \mathrm{ppm}_{\mathrm{pm}}=\right.$ 
161 were still performing well above chance (mean $=61.43, \mathrm{t}(77)=80.39, \mathrm{p}<.001,95 \% \mathrm{Cl}=$ $162[59.90 \%, 62.95 \%])$.

163 Measuring the difference in RTs on an ongoing task between PM and non-PM 164 trials is one of the primary methods for inferring PM strategy use ${ }^{8}$. A large difference in 165 OG RTs between PM and non-PM trials implies the use of proactive control on the PM 166 trials, whereas a small difference implies the use of reactive control on the PM trials.

167 Here, we found an interaction in OG RTs between condition (PM and non-PM trials) and 168 difficulty level $(1$ to 15$)(\beta$ interaction $=0.011, p<.001,95 \% \mathrm{Cl}=[0.010,0.013]$; Figure $2 \mathrm{~B})$. 169 At the hardest difficulty level, RTs were well below the response deadline of $1900 \mathrm{~ms}$ $170(\mathrm{t}(77)=38.401, \mathrm{p}<.001$, mean $\mathrm{RT}=1264 \mathrm{~ms}, 95 \% \mathrm{Cl}=[977 \mathrm{~ms}, 1551 \mathrm{~ms}])$,

171 demonstrating that participants were performing above floor. The difference in OG RT 172 between PM and non-PM trials (referred to as "PM cost" from here on out) was then 173 calculated for each participant at each difficulty level. These data were replotted in this 174 fashion, and they reveal that average PM costs varied systematically as a function of 175 OG task difficulty $\left(\beta_{\text {cost }}=-10.35, p<.001,95 \% \mathrm{Cl}=[-12.29,-8.42]\right.$; Figure $\left.2 \mathrm{C}\right)$. This 176 suggests that PM strategy shifted flexibly between proactive control and reactive control 177 as the OG task increased in difficulty, and vice versa. 
a

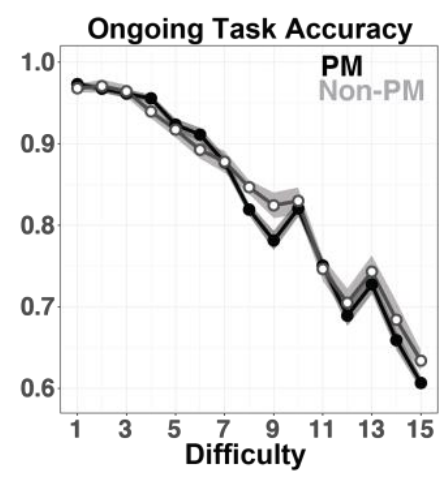

d

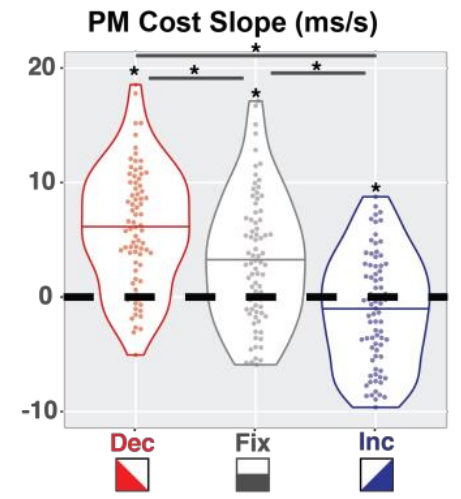

b

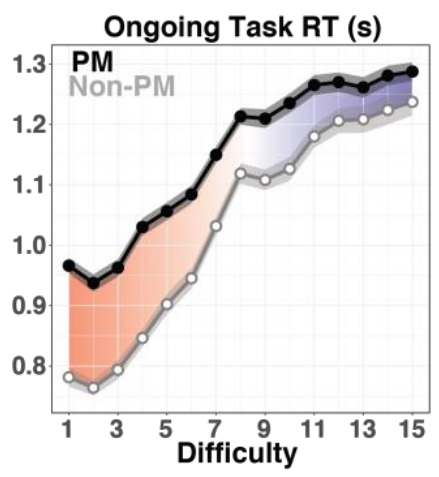

e

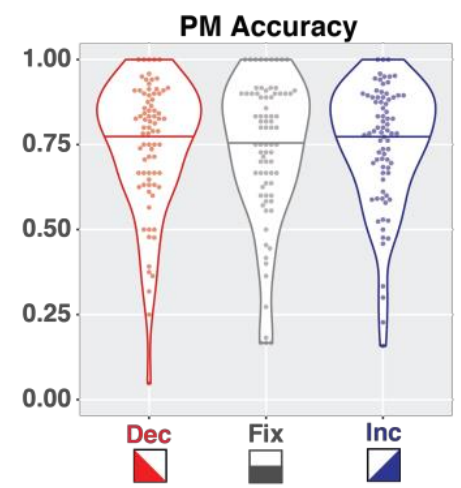

c

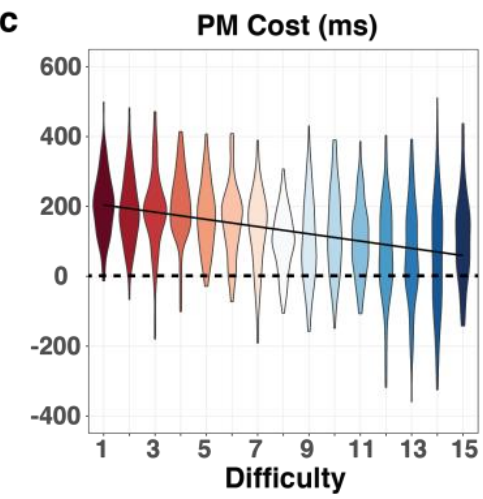

f

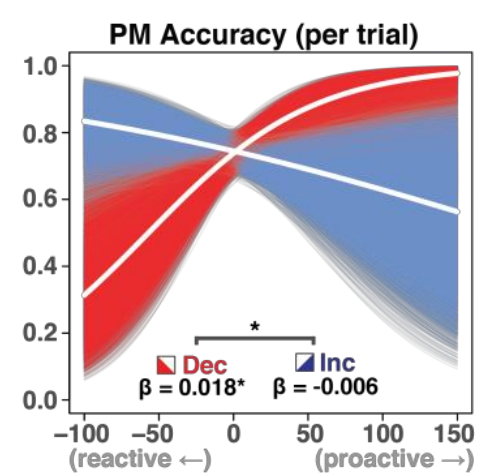

PM Cost Slope (ms/s)

Figure 2. Behavioral performance. a) Ongoing task accuracy across difficulties, error ribbons +/- 1 SEM. PM: dual-task trials with a PM intention; Non-PM: ongoing task only without a PM intention. b) Ongoing task RT (correct responses only) across difficulties, error ribbons +/- 1 SEM. c) PM cost (the difference between ongoing task RT for PM trials vs. Non-PM trials) was computed for each participant at every difficulty level. Violin plots represent the distribution of by-participant average costs at each difficulty. PM cost is highest at easy difficulty levels (dark red) and decreases as task difficulty increases (dark blue). d) PM cost slopes were calculated as the change in PM cost within each trial. Violin plots show the average within-trial PM cost slopes for decreasing (Dec), fixed (Fix), and increasing (Inc) trials across participants. * $p<.05$. e) PM accuracy for each participant across trial types. f) Logistic regression bootstrap analysis linking PM cost slope to PM accuracy for decreasing (red) and increasing (blue) trials. Each individual red/blue line shows the predicted relationship for each bootstrapped sample $(n=10,000)$. White lines reflect the fixed-effects relationship for the original sample. * $p<$ held within individual trials, we computed $1^{\text {st }}, 2^{\text {nd }}$, and $3^{\text {rd }}$ order polynomial regressions 
198 was bimodal (i.e., an all-or-none "flip" between proactive and reactive control), then 199 individual trials should be best fit by a $3^{\text {rd }}$ order polynomial. However, if the relationship was more fluid, then a $1^{\text {st }}$ or $2^{\text {nd }}$ order polynomial should fit the data better. Additionally,

201 if the data demonstrate a dramatic U-shaped or asymptotic curve, instead of a linear fit, 202 a $2^{\text {nd }}$ order polynomial should fit better than the $1^{\text {st }}$ order polynomial. We compared 203 Akaike information criterion values (AICc, with a correction for small sample sizes) for 204 each model for correct responses on each trial, and used Akaike weighting to compare 205 relative model fits. We found that a $1^{\text {st }}$ order polynomial (linear model) fit best for nearly 206 all trials $($ mean $=93.43 \%, 95 \% \mathrm{Cl}=[92.62 \%, 94.24 \%]$; Akaike weight $=0.873,95 \% \mathrm{Cl}=$ $207[0.865,0.880])$, compared to $2^{\text {nd }}$ order fits $($ mean $=5.72 \%, 95 \% \mathrm{Cl}=[4.99 \%, 6.45 \%]$; 208 Akaike weight $=0.111,95 \% \mathrm{Cl}=[0.104,0.119])$, or $3^{\text {rd }}$ order fits $($ mean $=0.85 \%, 95 \%$ $209 \mathrm{Cl}=[0.68 \%, 1.02 \%] ;$ Akaike weight $=0.016,95 \% \mathrm{Cl}=[0.012,0.019])$. This result 210 provides evidence that the engagement of control strategies changed fluidly and linearly 211 with changes in OG task demands within a PM trial. Next, we evaluated whether changes in PM cost within a trial were different for 213 increasing-, decreasing-, and fixed-difficulty trials. To do this, we computed a within-trial 214 measure of linear shift in PM cost from the beginning of the trial to the end of the trial, 215 which we shall refer to as PM cost slope. We found that PM cost slopes varied 216 systematically across trial types $(F(2,154)=47.02, p<.001$; Figure $2 D)$. PM cost 217 slopes were negative on increasing trials (mean $=-1.14 \mathrm{~ms} / \mathrm{s}, 95 \% \mathrm{Cl}=[-2.24,-0.42]$, $218 \mathrm{t}(77)=2.07, p=0.042$ ), and they were positive on decreasing trials ( $m e a n=6.03 \mathrm{~ms} / \mathrm{s}$, $21995 \% \mathrm{Cl}=[5.44,6.62], \mathrm{t}(77)=10.23, \mathrm{p}<.001)$ and fixed trials $($ mean $=3.34 \mathrm{~ms} / \mathrm{s}, 95 \% \mathrm{Cl}$ $220=[2.07,4.61], \mathrm{t}(77)=5.24, \mathrm{p}<.001)$. In this task design, a PM target appeared at the 
221 end of every PM trial. Thus, positive PM cost slopes (i.e., a shift towards proactive

222 control) on fixed difficulty trials likely arose from an increase in PM expectancy as each

223 trial progressed ${ }^{60,61}$. Expectancy should impact all trial types equally, and yet there were

224 meaningful differences between conditions. Planned pairwise comparisons revealed

225 that PM cost slopes increased step-wise from increasing trials to fixed trials $(F(77)=$

$22637.14, p<.001)$ and from fixed trials to decreasing trials $(F(77)=15.1, p<.001)$.

227

228 Prospective Memory Task Performance

229 On average, participants identified the PM target on three-quarters of the trials

230 (mean $\mathrm{PM}$ accuracy $=74.57 \%, 95 \% \mathrm{Cl}=[56.82 \%, 92.31 \%]$, with no differences in

231 accuracy across trial types $(F(2,154)=0.679, p=0.508$; Figure 1E). The false alarm

232 rate, defined as PM-target responses on probes where the target was not present, was

233 low (mean $=0.60 \%$ of probes, $95 \% \mathrm{Cl}=[-0.72 \%, 1.92 \%])$. Because PM accuracy was

234 stable across the dynamic trial types (increasing and decreasing difficulty trials), and

235 OG accuracy was not impacted by the presence of the PM task on these trials, we

236 concluded that participants were not sacrificing accuracy on one task in order to perform

237 the other. Therefore, any RT differences on the OG task during PM trials could

238 reasonably be interpreted as reflecting differences in strategy used to perform the PM

239 task, rather than a speed/accuracy tradeoff between the dual tasks.

241 Linking Shifts in PM Strategy to PM Performance

242

We reasoned that shifts in PM strategy might occur as individuals attempted to

243 preserve performance on the PM task while demands of the OG task shifted over time.

244 To evaluate this idea, we tested the relationship between PM cost slope and PM 
245 accuracy across all trials. We used bootstrapped logistic regression to relate these two

246 measures separately for increasing and decreasing trials (Figure 2F). On decreasing

247 trials, larger positive PM cost slopes (reflecting a shift towards proactive control) were

248 related to better PM performance $(\beta \mathrm{dec}=0.018, \mathrm{p}<.001,95 \% \mathrm{Cl}=[0.008,0.028])$. On

249 increasing trials, larger negative PM cost slopes (reflecting a shift towards reactive

250 control), were numerically related to better PM performance, but this relationship did not

251 reach statistical significance $\left(\beta_{\text {inc }}=-0.006, p=0.119,95 \% \mathrm{Cl}=[-0.015,0.004]\right)$.

252 Critically, there was an interaction of PM cost slope and trial type on PM accuracy, with

253 the direction of PM cost slope leading to different consequences on increasing vs.

254 decreasing trials $\left(\beta_{\text {interaction }}=0.024, p<.001,95 \% \mathrm{Cl}=[0.011,0.037]\right)$. On fixed trials

255 (data not shown), the relationship between PM cost slope and PM accuracy was

256 positive $\left(\beta_{\mathrm{fix}}=0.020, \mathrm{p}=0.003,95 \% \mathrm{Cl}=[0.006,0.033]\right)$, which was similar to

257 decreasing trials ( $\left.\beta_{\text {diff }}=0.001, p=0.423,95 \% \mathrm{Cl}=[-0.015,0.017]\right)$ but significantly

258 more positive than on increasing trials $\left(\beta_{\text {diff }}=0.025, p=0.001,95 \% \mathrm{Cl}=[0.009,0.041]\right)$.

259 The relationship between PM cost slope and PM accuracy survived after

260 controlling for other possible explanatory variables. Partial regression analyses

261 evaluated, one at a time, the impact of OG accuracy, OG RT, OG RT slope, and

262 average PM cost on the predictive link between PM cost slope and PM accuracy (see

263 supplementary materials). These control analyses confirmed that PM cost slope

264 explained a significant proportion of variance in PM accuracy, and did so to a greater

265 extent than all other variables.

266 This same by-trial relationship between PM cost slope and PM accuracy existed

267 across participants as well. Participants who on average showed larger shifts towards 
268 proactive control (more positive PM cost slopes) benefited more on decreasing trials, 269 and participants who showed larger shifts towards reactive control (more negative PM 270 cost slopes) benefited more on increasing trials ( $\beta$ interaction $=-0.018, p=0.002,95 \% \mathrm{Cl}=$

$271[-.013,-.024]$, data not shown). In summary, these results provide behavioral evidence

272 that individuals shifted PM strategy from moment to moment in response to changing

273 OG task demands. These shifts in cognitive control were adaptive because their

274 direction and magnitude were related to successful PM performance.

275

\section{Neural Measures of PM Intentions}

277 For the participants who performed this task in the MRI scanner $(\mathrm{N}=28)$, we 278 evaluated whether a neural measure of PM intention-related brain activity ${ }^{37}$ could 279 provide additional insight into the link between PM strategy selection and memory 280 performance. First, we identified regions that were significantly engaged by the PM task 281 above and beyond the OG task in isolation (Figure 3A; for more details see methods 282 and supplementary materials). These regions are consistent with previous literature on 283 PM intention maintenance ${ }^{36,37,39,41}$. From these brain regions, fMRI pattern classifiers 284 were used to quantify the degree of PM intention-related processing across each trial. 285 The strength of PM intention processing was operationalized as the difference in 286 classifier evidence for the PM-relevant category vs. the PM-irrelevant category (e.g., 287 "face - scene" for a face-target PM trial). Trained classifiers (for more details see 288 methods and supplementary materials) performed well above chance at predicting the 289 category of the current trial's PM target (classifier AUC for faces: 86.94, SE = 0.01; scenes $=88.54, \mathrm{SE}=0.01 ;$ non-PM trials: $83.76, \mathrm{SE}=0.01 ;$ and rest $=99.62, \mathrm{SE}=$ 
$\left.4.7^{*} 10^{-4}\right)$. Across trials, this neural measure varied systematically with OG task difficulty

292 (Figure 3B). The neural evidence of PM intention processing decreased as task demands increased $\left(\beta=-0.005, p<.001,95 \% \mathrm{Cl}=[-.008,-.002]\right.$, marginal $\left.\mathrm{r}^{2}=.039\right)$.
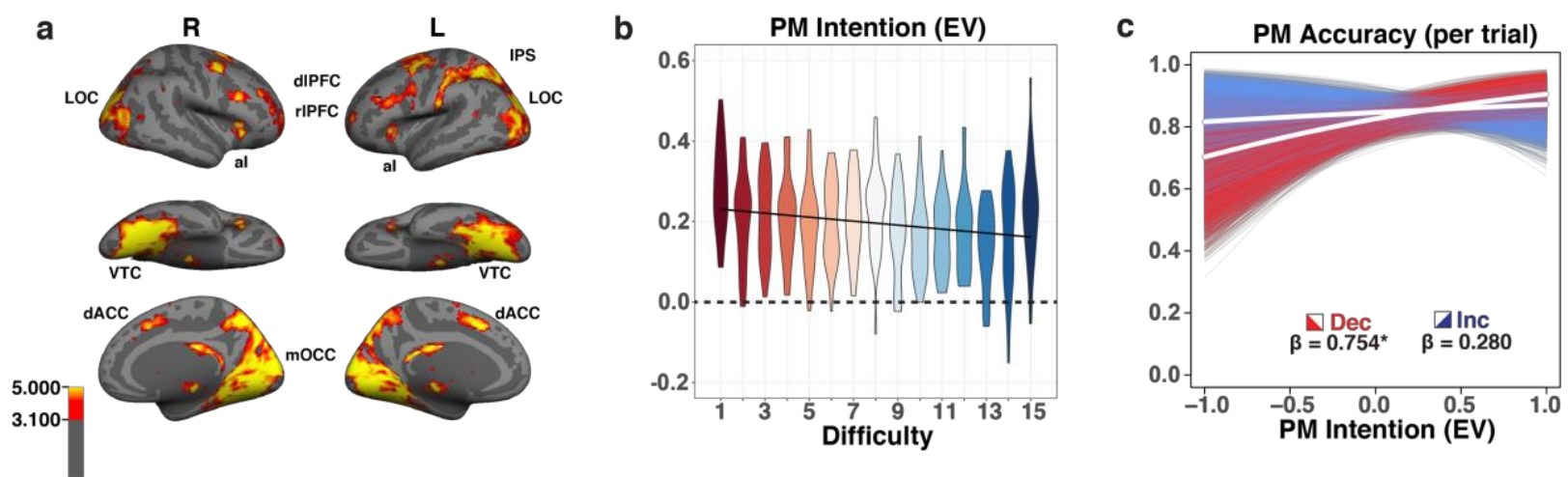

294

295

296

297

298

299

300

301

302

303

304

305

306

307

308

309

310

311

312

313

314

315

Figure 3. fMRI decoding of PM intentions. a) Brain regions significantly engaged by the addition of the PM task to the OG task (GLM contrast PM > Non-PM, FDR corrected at $p<.001)$. al $=$ anterior insular cortex, $\mathrm{dACC}=$ dorsal anterior cingulate cortex, $\mathrm{dIPFC}=$ dorsal lateral prefrontal cortex, IPS = Intraparietal sulcus, LOC = lateral occipital cortex, mOCC $=$ medial occipital cortex, rIPFC $=$ rostral lateral prefrontal cortex, VTC $=$ ventral temporal cortex. These regions were used as the initial feature mask to train and test fMRI pattern classifiers for PM intention-related activity. b) PM intention evidence (EV; the difference between classifier evidence for the PM target's category and the non-target category) was computed for each participant at every difficulty level, and group data is shown in violin plots. PM intention evidence was highest at easy difficulties (dark red) and lowest for the most difficult levels (dark blue). c) The relationship between PM intention evidence and PM accuracy was computed using bootstrapped logistic regression ( $n=10,000$ iterations) for decreasing (red) and increasing (blue) trials. ${ }^{*} p<.05$.

Within trials, however, the neural measure of PM intention processing did not vary systematically across timepoints (mean slope of PM intention evidence $=0.007$ $\mathrm{EV} / \mathrm{s}, 95 \% \mathrm{Cl}=[-0.002,0.016], \mathrm{t}(27)=1.57, \mathrm{p}=0.128)$. There were also no differences in by-trial PM intention evidence slopes across increasing, decreasing, and fixed difficulty trials $(F(2,54)=1.35, p=0.269)$. The stable level of $P M$ intention processing over the course of a single trial may be a measurement limitation due to the temporal sluggishness of the BOLD signal. Alternatively, it could also reflect the engagement of a 
316 prospective "retrieval mode"11,40,46, which has been described as a more sustained and

317 relatively inflexible component of proactive control that involves PM-items being held in

318 some prioritized state of working memory ${ }^{47}$. Therefore, we computed the average

319 classifier evidence for the PM intention on each trial and related this (rather than the 320 slope) to PM accuracy. A mixed-effect ANOVA confirmed that there were no overall 321 differences in average PM intention evidence across trial types $(F(2,54)=0.40, p=$ 322 0.670). This result was expected because increasing and decreasing trials spanned the 323 same range of difficulty levels (e.g., 1 to 15 vs. 15 to 1 ). We found that average PM 324 intention evidence correlated positively with PM accuracy on decreasing trials ( $\beta_{\mathrm{dec}}=$

$3250.754, p=.017,95 \% \mathrm{Cl}=[0.047,1.50]$; Figure $3 \mathrm{C}$ red $)$ and fixed trials $\left(\beta_{\mathrm{fix}}=0.976, p=\right.$ $326.039,95 \% \mathrm{Cl}=[-0.121,2.062]$; not shown $)$, but not on increasing trials $\left(\beta_{\text {inc }}=0.280, p=\right.$ $3270.255,95 \% \mathrm{Cl}=[-0.679,1.123]$; Figure $3 \mathrm{C}$ blue $)$. There were no reliable differences in 328 this statistic between increasing trials and either decreasing trials $\left(\beta_{\text {interaction }}=0.474, p=\right.$ $3290.199,95 \% \mathrm{Cl}=[-0.627,1.623])$ or fixed trials $($ interaction $=0.223, p=0.361,95 \% \mathrm{Cl}=[-$ $3301.119,1.473])$

332 Combining Behavioral \& Neural Measures to Predict PM Performance

333 We sought to test whether combining both the time-sensitive but indirect

334 behavioral metric of PM cost slope (putatively reflecting dynamic shifts in PM strategy) 335 and the coarser but more direct neural metric of PM intention evidence (putatively 336 reflecting sustained PM engagement) could improve our prediction of PM accuracy on a 337 trial by trial basis. There was no by-trial correlation between these measures (mean $r=$ $3380.02,95 \% \mathrm{Cl}=[-0.36,0.39], \mathrm{p}=0.92)$, suggesting that the two metrics could provide 
339 unique information about task performance. We performed a bootstrap analysis to

340 calculate the Akaike information criterion (AIC) values for models predicting PM

341 accuracy including all possible combinations of the predictors $P M$ cost slope, $P M$

342 intention evidence, and trial type (increasing/decreasing). We then selected the best

343 performing model that included (1) a neural and a behavioral metric, (2) only a

344 behavioral metric, or (3) only a neural metric. Next, we converted AIC scores for these

345 three models to Akaike weights (WAIC), allowing us to directly compare AIC values as

346 conditional probabilities ${ }^{62}$. The results show that the combined Behavior \& Neural model

347 (the full model including all main effects, all two-way interactions, and the three-way

348 interaction of PM cost slope, PM intention state, and trial direction) was the best model

349 (Wilcoxon median Akaike weight $=0.889$, Wilcoxon $95 \% \mathrm{Cl}=[0.883,0.896], p<.001$;

350 Figure 4A). This combined model was significantly more likely than either the best

351 Behavior-only model (Wilcoxon median ratio $=149.91$, wilcox $95 \% \mathrm{Cl}=[135.47$,

352165.63 ], $p<.001$ ) or the best Neural-only model (Wilcoxon median ratio $=4.5^{\star} 10^{9}$,

353 Wilcoxon $\left.95 \% \mathrm{Cl}=\left[3.9^{*} 10^{9}, 5.2^{*} 10^{9}\right], \mathrm{p}<.001\right)$.

a Predicting PM Acc. Model Fit

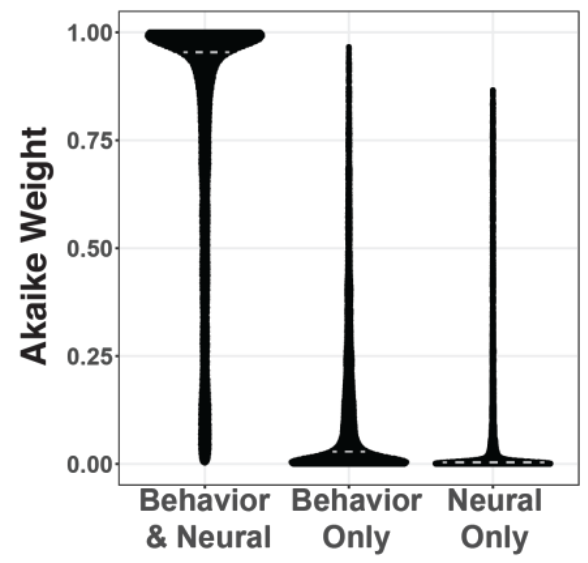

b Variance Explained in PM Acc.

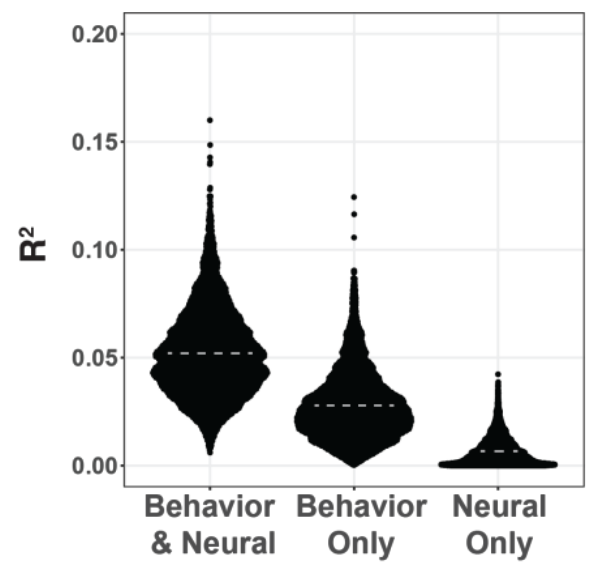


$357(n=10,000)$ to test model differences. a) Akaike weights (wAIC) across bootstrap 358 iterations for each model. b) Explanatory power of each model $\left(R^{2}\right)$ shown as 359 distributions across bootstraps. Medians are indicated by dashed grey lines.

We also found that on average the Behavior \& Neural model explained the most

362 variance in PM accuracy, with an $R^{2}$ value of approximately double that of the best

363 Behavior-only model $\left(R^{2}=.052\right.$ vs. .028, Figure 4B). Having found that the full

364 Behavioral \& Neural model was the best predictor of PM performance, we further

365 investigated the relationships in the model for reliability using the bootstrap approach.

366 We found that although including the three-way interaction term and multiple two-way

367 interaction terms improved the model fit, the only statistically reliable interaction was

368 that of $P M$ cost slope and trial direction $(\mathrm{p}=0.026)$. Additionally, we found that PM

369 intention evidence reliably had a positive relationship to PM accuracy on decreasing

370 difficulty trials (mean $=0.027, p=0.024$ ), but that relationship was not reliable on

371 increasing difficulty trials (mean $=-0.003, p=.395$ ). In summary, our model tests

372 revealed three main results: 1) Including both the neural and behavioral metrics of

373 proactive control improved prediction of PM accuracy over using either metric

374 independently. 2) PM cost slope was differentially predictive of PM accuracy for

375 increasing vs. decreasing trials, replicating the relationship from our behavior-only

376 analysis above (Figure 2F). 3) Higher levels of PM intention evidence were positively

377 related to PM performance on decreasing difficulty trials, but there was no reliable

378 relationship on increasing difficulty trials. See the supplementary material for further

379 information on the model fit analyses.

\section{Discussion}


This study investigated how navigating an environment with rapidly shifting

383

384

385

386

387

388

389

390

391

392

393

394

395

396

397

398

399

400

401

402

403

404

cognitive demands impacts how we remember to perform future actions. A delayedrecognition prospective memory $(\mathrm{PM})$ task was combined with a dynamic visual search ongoing (OG) task that varied in difficulty from moment to moment. When task difficulty was low, there was greater behavioral interference from the PM task ("PM cost": slower RTs in the OG task) and stronger neural representation of the PM intention ("PM intention evidence": classifier evidence for the PM target category in PM-sensitive brain regions). Both of these measures reflect components of proactive control ${ }^{5}$, and they were negatively correlated with OG task difficulty. The behavioral measure varied from moment to moment within a trial according to the task demands, whereas the neural measurement was stable within a given trial but varied across trials. Combining these behavioral and neural measures provided the best prediction of PM accuracy from trial to trial. Together, these results suggest that individuals dynamically adjusted their PM strategy in response to changes in environmental demands. Critically, we found that these shifts in PM strategy were adaptive because greater shifts (in the appropriate direction towards proactive or reactive control) were related to improvements in PM performance. The present results demonstrate that the ability to flexibly adjust cognitive control strategies, in response to changes in environmental demands, is an important contributor to successful execution of delayed intentions.

Our results are consistent with the dual mechanisms of cognitive control framework ${ }^{5,6}$ and the dynamic multiprocess framework of $\mathrm{PM}^{51,52}$. Both dual-mechanism frameworks posit that individuals can use two different methods of cognitive control to fulfill prospective intentions, and that they can flexibly adjust their control strategy in 
405 response to environmental factors, such as cognitive load or PM target expectancy.

406 However, neither framework formally describes whether that adjustment is a fluid

407 process or all-or-none "switch" between strategies. Here, our evidence suggests that

408 there are graded levels of control between proactive and reactive control that people 409 engage along a continuum.

410 We computed two distinct metrics of proactive strategy use: a time-sensitive

411 behavioral measure of PM cost, and a more tonic neural measure of PM intention

412 processing. The amount of PM costs (the behavioral measure) has been repeatedly

413 linked to levels of strategic monitoring for the PM intention ${ }^{8,10}$. Here, we found that

414 changes in the amount of PM costs over the course of a trial were associated with

415 better performance (Figure 2F). The DMC framework suggests that proactive control

416 would be favored on decreasing difficulty trials, when the OG task becomes

417 progressively easier, because attention and working memory resources should be

418 readily available to accomplish both the OG task and PM task successfully. On these

419 trials, the benefit of strategically monitoring for the PM intention may be worth the extra

420 cost incurred in RTs on the OG task. Our results support this idea, showing that when

421 participants reallocated cognitive resources to use proactive control on the PM task

422 (positive PM cost slopes within a trial), PM performance improved.

423 Conversely, on increasing difficulty trials, when the OG task becomes

424 progressively more difficult, the DMC framework predicts that reactive control would be

425 favored. When there are not sufficient resources to allocate to both the PM task and the

426 OG task, the ability to strategically monitor for the PM intention is compromised, and

427 reliance on this process may lead to deficits in PM performance. Such deficits may arise 
428 from interference in working memory caused by failed attempts to maintain a robust

429 representation of the PM target in the face of distractors, a reduced ability to shift

430 attention between the two tasks in order to strategically monitor for the PM cue

431 effectively, or a reduced ability to perform the PM intention even after noticing a

432 prospective cue $e^{49,63,64}$. Consistent with these ideas, we found that when participants did

433 not divert cognitive resources away from the PM task as OG task difficulty increased

434 (i.e., when PM cost slopes were positive on these trials ), PM performance suffered.

435 Guynn's two-component model of proactive control ${ }^{11,46}$, dissociates a flexible,

436 strategic monitoring component from a more tonic component referred to as the "PM-

437 retrieval mode", which involves sustained maintenance of the PM task set. In the

438 present study, we believe that the behavioral measure of PM cost likely reflects the

439 strategic monitoring component of this model, whereas the neural measure of PM

440 intention evidence likely reflects the PM-retrieval mode. Our results implicated neural

441 regions associated with working memory ${ }^{57-59}$, including the anterior prefrontal cortex,

442 with this neural measure. However, we found that regions known to support perception

443 and working memory for the specific PM intentions used here (i.e., the ventral temporal

444 cortex $^{65,66}$ for face and scene stimuli) were most important for identifying PM intention

445 maintenance (as identified by a surface-based searchlight analysis ${ }^{67,68}$; see the

446 supplementary materials). We found that on decreasing difficulty trials (which, according

447 to the behavioral analysis, favor a shift towards proactive control), PM intention

448 maintenance was positively correlated with memory performance, but on increasing

449 trials (which favor a shift towards reactive control) it was not (Figure 3C). Although

450 individuals sometimes exhibited a high level of PM readiness, this did not influence task 
451 performance in situations that favored reactive control. These results are in line with

452 previous research suggesting that PM intention maintenance could be beneficial to PM

453 performance in some situations, but was not necessary for successful realization of PM 454 intentions in all situations ${ }^{40}$.

455 The DMPV framework ${ }^{51,52}$ also posits that in contexts where PM occurrence is 456 highly probable, individuals are biased towards and benefit from using proactive control.

457 In our study, participants knew that a PM target would reappear relatively soon after it

458 was introduced (between 2-30s later). On trials with fixed difficulty, we found a

459 consistent increase in PM costs across each trial (positive PM cost slopes), and greater

460 increases in cost were related to better PM performance. This indicates a beneficial, 461 perhaps default, shift towards proactive control in this paradigm for which there is an 462 increasing probability of a PM event throughout each trial. Shifts towards proactive 463 control were even stronger (and beneficial for performance) on decreasing difficulty 464 trials as more cognitive resources became available over time. In contrast, participants 465 shifted away from proactive control on increasing difficulty trials, as cognitive resources 466 were being consumed more and more by ongoing demands over time.

467 An alternative explanation for the present results is that shifts in PM cost may not 468 reflect shifts between proactive and reactive control strategies per se, but rather shifts 469 between stronger and weaker levels of proactive control. Unitary models of PM such as 470 the preparatory attention and memory "PAM" theory ${ }^{10}$ propose that successfully fulfilling 471 prospective intentions relies on some level of proactive preparation in all situations.

472 However, we believe this interpretation of our data is less likely than the dual-

473 mechanisms account. According to the PAM model, we should expect extremely poor 
474 PM performance when evidence of proactive control is absent, and this was not the 475 case. On trials when the PM target appeared while PM costs were high, detection 476 accuracy of the PM target was quite good (mean $=76.62 \%, \mathrm{SEM}=1.94 \%$; average $\mathrm{N}=$ 47737.7 trials/participant). However, on trials where PM costs were absent (indicating no 478 evidence of proactive control) when the PM target appeared, accuracy was worse $(\mathrm{t}(76)$ $479=4.684, p<.001)$, but still relatively good and well above floor $($ mean $=67.81 \%$, SEM $=$ $4802.67 \%, \mathrm{~N}=12.1$ trials/participant). PM performance was also strong on trials where the 481 neural measure of PM intention evidence was absent ( mean $=81.1 \%, \mathrm{SE}=3.2 \%$, 482 average $\mathrm{N}=11.5$ trials/participant), and also on trials where both $\mathrm{PM}$ cost and $\mathrm{PM}$ 483 intention evidence were absent $($ mean $=70.5 \%, \mathrm{SE}=6.5 \%$, average $\mathrm{N}=2.8$

484 trials/participant). Incidentally, the link between PM cost and PM accuracy in the present 485 study closely replicates prior work from our lab ${ }^{37}$ where PM accuracy was $66.0 \%$ (SEM: $4864.1 \%$ ) when participants were biased towards reactive control, and $71.2 \%$ (SEM: $3.0 \%$ ) 487 when participants were biased towards proactive control.

One limitation of the current study is its reliance on the behavioral PM cost 489 measure to infer PM strategy use. While this has become a standard approach, PM cost 490 is nonetheless an indirect measure of PM strategy, the underlying source of which is still 491 under debate ${ }^{53,69-71}$. We sought to complement this indirect measure with a more direct 492 measure of PM intention processing using $\mathrm{fMRI}$ pattern classifiers to track PM intention 493 maintenance. However, fMRI is sluggish and not ideal to observe time-sensitive shifts in 494 neural coding. It is possible that our neural measure incorporates aspects of both PM495 intention maintenance as well as strategic monitoring. However, these neural measures 496 were not correlated with PM cost slopes, which are believed to reflect changes in 
497 monitoring. In addition, previous work has found that in contexts where participants are

498 biased towards reactive control, the level of monitoring is not related to PM

499 performance ${ }^{18,72}$, however it is related to PM performance when participants are biased

500 towards proactive control $37,53,72$.

501 We find that our behavioral and neural metrics provide complementary but

502 independent information about PM performance, approximately doubling our model's

503 predictive power when the neural measure of PM intention maintenance was combined

504 with the behavioral metric of PM cost slope (Figure 4B). This result suggests that our

505 measures are capturing different components of proactive control, though the current

506 design does not specify the relative contribution of strategic monitoring versus PM

507 intention maintenance. A future direction will be to use a more time-sensitive neural

508 measure like EEG (electroencephalogram) and eye-tracking to measure active

509 maintenance of PM intentions in dynamic environments, as well as to better identify

510 late-retrieval mechanisms that are characteristic of reactive control strategy use.

511 In conclusion, here we developed a novel dual-task paradigm to show that

512 people solve prospective memory problems by flexibly shifting between proactive

513 control and reactive control in response to changes in ongoing cognitive demands. We

514 found evidence for two different components of proactive control - strategic monitoring,

515 measured behaviorally, and PM intention maintenance, measured neurally - which

516 independently fluctuate and contribute to PM performance. These shifts were adaptive

517 in that adjustments of control towards the strategy favored for a given situation (e.g.,

518 shifting towards proactive control when demands decreased across time) led to better

519 PM performance. These results extend dual mechanism accounts of PM by 
520 demonstrating that cognitive flexibility (i.e., adapting cognitive control strategies to the

521 environment) is beneficial for remembering to perform future intentions.

522

\section{Methods}

524 In this experiment we had 78 healthy young adult participants (28 neural sample,

$52517 \mathrm{~F}$, mean age $=21.8 ; 50$ behavioral sample, $31 \mathrm{~F}$, mean age $=19.2$ ), perform an

526 ongoing visual search task with an embedded PM task (Figure 1). The ongoing task

527 here was a visual-search task ("OG task") where participants searched for a specific

528 target arrow on a circular array of oriented arrows (Figure 1A). We chose this as the

529 OG task due to the ability to systematically and parametrically manipulate task difficulty

530 by adjusting distractor parameters along a continuum ${ }^{73}$. The ongoing task target arrow

531 was always a rightward facing horizontal arrow $(\Rightarrow)$, which was present on only a

532 randomly selected half of all trials, located in one of 10 semi-randomly selected possible

533 locations around the circle. Participants were instructed to search for the target on each

534 display and use their right hand to press "1" for present or "2" for absent. Target arrow

535 location was counterbalanced between the top and bottom half of the screen. Non-

536 target (distractor) arrows appeared in set positions around the circular array, oriented

537 within some distribution of angles determined by the current task difficulty setting. OG

538 task difficulty was manipulated on each probe by adjusting two parameters controlling

539 the orientation of the distractor arrows: their minimum similarity to the target, and their

540 similarity to other distractors. A minimum angular distance for distractor-to-target

541 similarity was set to either $5,15,25,45,65$, or 75 degrees. For distractor-to-distractor

542 similarity, the maximum variance from the minimum angular distance was set to either 
54310,20 , or 40 degrees. The factorial combination of these parameters (excluding any

544 combination where minimum plus variance could exceed the $90^{\circ}$ vertical plane) created

54515 difficulty conditions that gradually and monotonically changed in difficulty (see

546 supplementary materials for OG task difficulty pilot study results). On every search

547 display, each distractor arrow had a $50 \%$ chance of being randomly flipped across the

548 horizontal plane and a $50 \%$ chance of being flipped across the vertical plane, so that

549 distractor arrows could vary from horizontal across either $5-175^{\circ}$ or $185-355^{\circ}$. In order to

550 minimize uncontrolled pop-out effects, we ensured that no distractor arrow in the array

551 was within 5 degrees of an arrow that appeared in the same location on the circle during

552 the previous display. This manipulation ensured that each visual-search array was a

553 completely randomized new array of possible targets and distractors.

554

\section{PM Task Stimuli}

556 Colored images of unfamiliar faces and unfamiliar scenes were gathered from

557 various in-house and online sources. These images were controlled for valence and

558 familiarity. Of those images, 230 (115 faces, 115 scenes) were selected for use in this

559 experiment. For each participant, 40 faces and 40 scenes were randomly selected to

560 serve as the PM targets, 75 faces and 75 scenes were used as distractors. PM target

561 images did not appear as distractors and were used on one trial only. Distractor images

562 never reappeared within the same trial, but later reappeared on subsequent trials (mean

563 exposures per distractor $=14, \min =6, \max =20$ ).

564

565 PM Task Description 
Participants completed six blocks (five in the fMRI sample) of 20 trials each.

567 Blocks were separated by brief rest periods, the length of which was determined by the

568 participant. Five trials in each block were "catch trials" (containing fewer than 8 probes),

569 which were included to keep participants engaged at the beginning of each trial. These

570 short trials were included for the sole purpose of making sure that individuals did not

571 ignore the PM-task component on early-trial probes. Performance on these catch trials

572 was ignored, leaving 15 experimental ("real") trials per block. PM and non-PM trials

573 were randomly intermixed within each block, with one-third of all trials being non-PM

574 trials, and the other two-thirds were equally split between face- or scene-target PM

575 trials. OG task difficulty was manipulated in five conditions: it could either (1) increase

576 starting at the easiest difficulty (level 1), (2) increase starting at the median difficulty

577 (level 8), (3) decrease starting at the hardest difficulty (level 15), (4) decrease starting at

578 the median difficulty, or (5) remain fixed at the median difficulty. For the main analyses

579 reported here, the first two conditions were combined as "increasing" trials, the second

580 two conditions were combined as "decreasing" trials, and the fifth condition was referred

581 to as "fixed". Changes in difficulty occurred at the rate of one shift in difficulty level per

582 probe until either the end of the trial or until a difficulty endpoint was reached. Each of

583 the five difficulty types occurred three times throughout each block in pseudo-random

584 order. Fifteen "real" trials corresponding to the $3 \times 5$ combinations of PM type

585 (face/scene/non-PM) and OG difficulty type occurred once per block. Performance on

586 face and scene trials was similar, so to increase statistical power, these trials were

587 collapsed for subsequent analyses. The difficulty types for catch trials were 
588 counterbalanced across the entire experiment. For more specifics about task design 589 and the difficulty manipulation, see supplementary materials.

590 Each trial began with the presentation of the PM target (a face, a scene, or no 591 target) for 3-sec, followed by a 1-sec fixation cross. For non-PM trials, participants saw 592 a yellow null $(\varnothing)$ sign in lieu of a face or scene. For PM trials, participants were informed 593 that the PM target shown was only relevant for the current trial. After target 594 presentation, participants saw a series of 1-15 probes per trial. Every probe contained a 595 visual-search array in the center of the screen, with one face and one scene (each of 596 size $9.5^{\circ} \times 9.5^{\circ}$ in visual angle) vertically aligned with the center of the images placed $59711.5^{\circ}$ above or below the search array. Each probe was on the screen for $2 \mathrm{sec}$, during 598 which the participants were allowed 1.9s to respond to the presence or absence of the 599 horizontal arrow in the OG task, or to indicate whether the PM target had reappeared. 600 They were instructed to equally weight the importance of both tasks, and only one 601 response (to either the OG task or the PM task) was allowed per probe ("task-switch" 602 approach $^{74}$ ). Trial lengths were predetermined and pseudo-randomized so that every 603 participant had the same number of total probes for face-target, scene-target, and non604 PM trials. Scene and face locations were randomized on each probe, and faces and 605 scenes appeared on the top or bottom of the display with equal probability. Visual 606 feedback was presented immediately following each probe response, in the form of the 607 arrows turning green for correct OG responses, turning red for incorrect OG responses, 608 or a yellow border surrounding the screen for PM false alarms. Probe feedback 609 remained on screen for the remaining duration of each 2-sec probe. The $1.9 \mathrm{~s}$ response 
610 deadline (of $2 \mathrm{~s}$ total probe length) ensured that some time (minimum $100 \mathrm{~ms}$ ) was

611 always devoted to feedback on every probe.

612 On trials with a PM target presented at the beginning of the trial ("PM trials"),

613 participants performed both tasks as described above. On trials with no PM target

614 presented ("non-PM trials"), participants were instructed to ignore the face and scene

615 images and focus solely on the OG task. The PM target reappeared only once per trial,

616 and its reappearance always marked the end of the trial. After the final probe of each

617 trial, participants were given feedback on the PM task in the form of a green border

618 appearing around the edge of the screen for correct PM responses and a red border for

619 missed PM targets. This feedback (or a blank screen for non-PM trials) remained for $2 \mathrm{~s}$

620 and was followed by a 6-s ITI with a fixation cross on the screen before the next trial

621 began.

622

\section{MRI acquisition and preprocessing}

624 MRI data were acquired on a 3.0-T Siemens Skyra MRI scanner with a 32-

625 channel head coil. Whole brain, high-resolution anatomical images were collected for 626 registration and parcellation using a T1-weighted MPRAGE sequence (repetition time

$627(\mathrm{TR})=1900 \mathrm{~ms}$, echo time $(\mathrm{TE})=2.43 \mathrm{~ms}$, flip angle $=9^{\circ}$, field of view $(\mathrm{FOV})=$

$628256 \times 256 \times 192,1 \mathrm{~mm}$ isotropic voxels). Functional images were acquired using a T2*-

629 weighted multiband accelerated EPI pulse sequence $(T R=2 s, T E=29 \mathrm{~ms}$, flip angle $=$

$63078^{\circ}, \mathrm{FOV}=76 \times 76$, slice-thickness $=3 \mathrm{~mm}$, multiband factor $=2$, number of slices $=48$, no

631 gap). Following shim correct at the beginning of each scan session, a B0 field-map with

632 the same slice prescription as the functional data was acquired. 
633

634

635

636

637 images were first slice time corrected using FSL's slicetimer function. Then, functional

638 runs were normalized to the third run of the main task (middle run) using a combination

639 of within-run motion correction, rigid and affine registration, and field unwarping

640 processes from FSL and ANTs. Non-linear registration, via antsRegistrationSyn, was

641 then used to correct for between run differences. Lastly, high pass filtering (128s) was

642 applied to the images. Multivariate pattern analyses were then performed within this

643 registered subject space, independently for each participant (for more MVPA details,

644 see the supplementary materials).

645 For the univariate GLM analysis, rigid and affine transformations were used to

646 register the functional scans to the high-resolution anatomical, and then non-linear

647 transformations were applied to normalize runs to the MNI-template. These images

648 were spatially smoothed (5 mm gaussian), but no further preprocessing was performed

649 before using FSL FEAT for modeling. The model included separate regressors for the

650 PM target presentation, PM trial probes 1 to $n-1$, probe $n$ (when the PM target

651 reappeared in PM trials), non-PM trial probes 1 to $n$, trial-feedback, and rest. Six motion

652 parameters, extracted using FSL's MCFLIRT, were included as confound regressors.

653 FSL's FEAT was used to identify voxels that were more responsive on PM trials

$654(\mathrm{PM}+\mathrm{OG})$ than on non-PM (OG-only) trials (cluster correction, $\mathrm{p}<.001$; see

655 supplementary materials). 
This group level results map was then individually transformed from standard

657 space into subject functional space using the inverse normalization parameters used for

658 mapping subject functional to anatomical scans and the anatomical scans to the MNI

659 template, and then used as an initial feature selection mask for multivariate pattern

660 classification analysis. A classifier was trained (using the Princeton MVPA toolbox,

$661 \mathrm{https://github.com/princetonuniversity/princeton-mvpa-toolbox)} \mathrm{to} \mathrm{distinguish} \mathrm{between}$

662 TRs with face PM targets, scene PM targets, no-PM target (OG task only), and rest. For

663 each k-fold iteration, we used data from the three face/scene/rest localizer training

664 blocks and two OG/rest localizer training blocks (see supplementary materials), as well

665 as four of the five PM task blocks for further feature selection and training. One PM task

666 block was held out on each fold for testing.

For all analyses involving RTs, we excluded any responses faster than $300 \mathrm{~ms}$.

668 This criterion is in line with previous work ${ }^{69,79}$ and was used in order to exclude late 669 responses carried over from the preceding response window. Because this paradigm

670 involves long PM trials (mean 28s, range 8-36s), it produced few behavioral PM reports

671 per participant. Therefore, to increase statistical power for this analysis, we performed a

672 non-parametric bootstrap analysis using data sampled from all participants. On each

673 bootstrap iteration $(n=10000)$ of this analysis, 78 participants were selected at random

674 with replacement and combined to make one super subject. We used logistic regression

675 to test the relationship between PM accuracy and PM cost slope and/or PM intention

676 evidence on the different trial types. The stability of the effects across all iterations was

677 analyzed to assess population-level reliability. 


\section{Acknowledgements}

680

S.R.K., A.M., and J.L.P. conceived the study. S.R.K. and J.L.P. designed the

681 experiment and wrote the paper. S.R.K. and K.H. collected and analyzed data under the

682 supervision of J.L.P. The authors would like to thank Bettina Bustos, Stephanie

683 Jeanneret, and Robaa Al-Najar for their contributions to data collection, as well as Neal

684 Morton for help with MRI data preprocessing. This work was supported by funds from

685 the University of Texas System STARs Program, a UT BRAIN seed grant, and NIH

686 grant R01 EY028746 awarded to J.L.P.

687

688 References

689 1. Gratton, G., Cooper, P., Fabiani, M., Carter, C. S. \& Karayanidis, F. Dynamics of cognitive

690 control: Theoretical bases, paradigms, and a view for the future. Psychophysiology 55,

691 e13016 (2018).

692 2. Dismukes, R. K. Prospective Memory in Workplace and Everyday Situations. Curr. Dir.

693 Psychol. Sci. 21, 215-220 (2012).

694 3. Crovitz, H. F. \& Daniel, W. F. Measurements of everyday memory: Toward the prevention of

695 forgetting. Bull. Psychon. Soc. 22, 413-414 (1984).

696 4. Kliegel, M. \& Martin, M. Prospective memory research: Why is it relevant? Int. J. Psychol.

$69738,193-194(2003)$.

698 5. Braver, T. S. The variable nature of cognitive control: a dual mechanisms framework. Trends

699 Cogn. Sci. 16, 106-113 (2012). 
700 6. Braver, T. S., Gray, J., R. \& Burgess, Z. Explaining the many varieties of working memory

701 variation: dual mechanisms of cognitive control. in Variation in working memory 142-214

702 (Oxford University Press, 2007).

703 7. Chiew, K., S. \& Braver, T., S. Context Processing and Cognitive Control. in The Wiley

704 Handbook of Cognitive Control 143-166 (John Wiley \& Sons Ltd., 2017).

705 8. Einstein, G. O. \& McDaniel, M. A. Prospective Memory: Multiple Retrieval Processes. Curr.

706 Dir. Psychol. Sci. 14, 286-290 (2005).

707 9. McDaniel, M. A. \& Einstein, G. O. Strategic and automatic processes in prospective memory

708 retrieval: a multiprocess framework. Appl. Cogn. Psychol. 14, S127-S144 (2000).

709 10. Smith, R. E. The cost of remembering to remember in event-based prospective memory:

710 Investigating the capacity demands of delayed intention performance. J. Exp. Psychol.

711 Learn. Mem. Cogn. 29, 347-361 (2003).

712 11. Guynn, M. J. A two-process model of strategic monitoring in event-based prospective

713 memory: Activation/retrieval mode and checking. Int. J. Psychol. 38, 245-256 (2003).

714 12. Brewer, G. A., Knight, J. B., Marsh, R. L. \& Unsworth, N. Individual differences in event-

715 based prospective memory: Evidence for multiple processes supporting cue detection.

716 Mem. Cognit. 38, 304-311 (2010).

717 13. Einstein, G. O. \& McDaniel, M. A. Prospective memory and what costs do not reveal about

718 retrieval processes: A commentary on Smith, Hunt, McVay, and McConnell (2007). J. Exp.

719 Psychol. Learn. Mem. Cogn. 36, 1082-1088 (2010). 
720 14. Marklund, P. \& Persson, J. Context-dependent switching between proactive and reactive 721 working memory control mechanisms in the right inferior frontal gyrus. Neurolmage 63, $722 \quad 1552-1560(2012)$.

723 15. McDaniel, M., A. \& Einstein, G., O. Prospective memory: an overview and synthesis of an 724 emerging field. (SAGE Publications, 2007).

725 16. Cohen, A.-L. Number of cues influences the cost of remembering to remember. Mem.

726 Cognit. 36, 149-156 (2008).

727 17. Smith, R. E., Hunt, R. R., McVay, J. C. \& McConnell, M. D. The cost of event-based

728 prospective memory: Salient target events. J. Exp. Psychol. Learn. Mem. Cogn. 33, 734-746

729 (2007).

730 18. Harrison, T. L. \& Einstein, G. O. Prospective memory: Are preparatory attentional processes 731 necessary for a single focal cue? Mem. Cognit. 38, 860-867 (2010).

732 19. Knight, J. B. et al. An observation on the spontaneous noticing of prospective memory 733 event-based cues. J. Exp. Psychol. Learn. Mem. Cogn. 37, 298-307 (2011).

734 20. Rummel, J. \& Meiser, T. The role of metacognition in prospective memory: Anticipated task 735 demands influence attention allocation strategies. Conscious. Cogn. 22, 931-943 (2013).

736 21. Scullin, M. K., McDaniel, M. A. \& Einstein, G. O. Control of cost in prospective memory: 737 Evidence for spontaneous retrieval processes. J. Exp. Psychol. Learn. Mem. Cogn. 36, 190$738203(2010)$.

739 22. Scullin, M. K., McDaniel, M. A., Shelton, J. T. \& Lee, J. H. Focal/nonfocal cue effects in 740 prospective memory: Monitoring difficulty or different retrieval processes? J. Exp. Psychol. $741 \quad$ Learn. Mem. Cogn. 36, 736-749 (2010). 
742 23. Harrison, T. L., Mullet, H. G., Whiffen, K. N., Ousterhout, H. \& Einstein, G. O. Prospective

743 memory: Effects of divided attention on spontaneous retrieval. Mem. Cognit. 42, 212-224

744 (2014).

745 24. Marsh, R. L. \& Hicks, J. L. Event-Based Prospective Memory and Executive Control of

746 Working Memory. J. Exp. Psychol. Learn. Mem. Cogn. 24, 336-349 (1998).

747 25. Rose, N. S., Rendell, P. G., McDaniel, M. A., Aberle, I. \& Kliegel, M. Age and individual

748 differences in prospective memory during a 'Virtual Week': The roles of working memory,

749 vigilance, task regularity, and cue focality. Psychol. Aging 25, 595-605 (2010).

750 26. Smith, R. E. \& Bayen, U. J. The Effects of Working Memory Resource Availability on

751 Prospective Memory: A Formal Modeling Approach. Exp. Psychol. 52, 243-256 (2005).

752 27. Schnitzspahn, K. M., Stahl, C., Zeintl, M., Kaller, C. P. \& Kliegel, M. The role of shifting,

753 updating, and inhibition in prospective memory performance in young and older adults.

754 Dev. Psychol. 49, 1544-1553 (2013).

755 28. Henry, J. D., MacLeod, M. S., Phillips, L. H. \& Crawford, J. R. A Meta-Analytic Review of

756 Prospective Memory and Aging. Psychol. Aging 19, 27-39 (2004).

757 29. Ihle, A., Hering, A., Mahy, C. E. V., Bisiacchi, P. S. \& Kliegel, M. Adult age differences,

758 response management, and cue focality in event-based prospective memory: A meta-

759 analysis on the role of task order specificity. Psychol. Aging 28, 714-720 (2013).

760 30. Kliegel, M., Jäger, T. \& Phillips, L. H. Adult age differences in event-based prospective

761 memory: A meta-analysis on the role of focal versus nonfocal cues. Psychol. Aging 23, 203-

$208(2008)$ 
763 31. Park, D. C., Hertzog, C., Kidder, D. P., Morrell, R., W. \& Mayhorn, C., B. Effect of Age on

764 Event-Based and Time-Based Prospective Memory. Psychol. Aging 12, 314-327 (1997).

765 32. Smith, R. E. \& Hunt, R. R. Prospective memory in young and older adults: The effects of task

766 importance and ongoing task load. Aging Neuropsychol. Cogn. 21, 411-431 (2014).

767 33. Vogels, W. W. A., Dekker, M. R., Brouwer, W. H. \& de Jong, R. Age-Related Changes in

768 Event-Related Prospective Memory Performance: A Comparison of Four Prospective

769 Memory Tasks. Brain Cogn. 49, 341-362 (2002).

770 34. Bugg, J. M. Evidence for the sparing of reactive cognitive control with age. Psychol. Aging

$77129,115-127(2014)$.

772 35. Scullin, M. K., Bugg, J. M., McDaniel, M. A. \& Einstein, G. O. Prospective memory and aging:

773 preserved spontaneous retrieval, but impaired deactivation, in older adults. Mem. Cognit.

$774 \quad 39,1232-1240$ (2011).

775 36. Beck, S. M., Ruge, H., Walser, M. \& Goschke, T. The functional neuroanatomy of

776 spontaneous retrieval and strategic monitoring of delayed intentions. Neuropsychologia 52,

$777 \quad 37-50(2014)$.

778 37. Lewis-Peacock, J. A., Cohen, J. D. \& Norman, K. A. Neural evidence of the strategic choice

779 between working memory and episodic memory in prospective remembering.

$780 \quad$ Neuropsychologia 93, 280-288 (2016).

781 38. Reynolds, J. R., West, R. \& Braver, T. Distinct Neural Circuits Support Transient and

782 Sustained Processes in Prospective Memory and Working Memory. Cereb. Cortex 19, 1208-

$783 \quad 1221$ (2009). 
784

785

786

788

789

790

791

792

793

794

795

796

797

798

799

800

801

802

803

804

805

39. McDaniel, M. A., LaMontagne, P., Beck, S. M., Scullin, M. K. \& Braver, T. S. Dissociable Neural Routes to Successful Prospective Memory. Psychol. Sci. 24, 1791-1800 (2013).

40. Cona, G., Bisiacchi, P., S. \& Moscovitch, M. The Effects of Focal and Nonfocal Cues on the Neural Correlates of Prospective Memory: Insights From ERPs. Cereb. Cortex 24, 2630-2646 (2014).

41. Cona, G., Scarpazza, C., Sartori, G., Moscovitch, M. \& Bisiacchi, P. S. Neural bases of prospective memory: A meta-analysis and the "Attention to Delayed Intention" (AtoDI) model. Neurosci. Biobehav. Rev. 52, 21-37 (2015).

42. West, R., Bowry, R. \& Krompinger, J. The effects of working memory demands on the neural correlates of prospective memory. Neuropsychologia 44, 197-207 (2006).

43. West, R., McNerney, M. W. \& Travers, S. Gone but not forgotten: The effects of cancelled intentions on the neural correlates of prospective memory. Int. J. Psychophysiol. 64, 215225 (2007).

44. Scullin, M. K., Bugg, J. M. \& McDaniel, M. A. Whoops, I did it again: Commission errors in prospective memory. Psychol. Aging 27, 46-53 (2012).

45. Anderson, F. T., McDaniel, M. A. \& Einstein, G. O. Remembering to Remember: An Examination of the Cognitive Processes Underlying Prospective Memory is. in Learning and Memory: A Comprehensive Reference 451-463 (Elsevier, 2017). doi:10.1016/B978-0-12809324-5.21049-3

46. Guynn, M., J. Theory of Monitoring in Prospective Memory Instantiating a Retrieval Mode and Periodic Target Checking. in Prospective Memory: Cognitive, Neuroscience, Developmental, and Applied Perspectives 53-76 (Psychology Press, 2008). 
806 47. Underwood, A. G., Guynn, M. J. \& Cohen, A.-L. The Future Orientation of Past Memory: The 807 Role of BA 10 in Prospective and Retrospective Retrieval Modes. Front. Hum. Neurosci. 9, 1$80812(2015)$

809 48. Whitehead, P., S. \& Egner, T. Cognitive Control Over Prospective Task-Set Interference. J. 810 Exp. Psychol. Hum. Percept. Perform. 44, 741-755 (2018).

811 49. Ballhausen, N., Schnitzspahn, K. M., Horn, S. S. \& Kliegel, M. The interplay of intention 812 maintenance and cue monitoring in younger and older adults' prospective memory. Mem.

813 Cognit. 45, 1113-1125 (2017).

814 50. Gilbert, S. J., Hadjipavlou, N. \& Raoelison, M. Automaticity and Control in Prospective 815 Memory: A Computational Model. PLoS ONE 8, e59852 (2013).

816 51. Scullin, M. K., McDaniel, M. A. \& Shelton, J. T. The Dynamic Multiprocess Framework:

817 Evidence from prospective memory with contextual variability. Cognit. Psychol. 67, 55-71 818 (2013).

819 52. Shelton, J. T. \& Scullin, M. K. The Dynamic Interplay Between Bottom-Up and Top-Down 820 Processes Supporting Prospective Remembering. Curr. Dir. Psychol. Sci. 26, 352-358 (2017).

821 53. Ball, B. H., Brewer, G. A., Loft, S. \& Bowden, V. Uncovering continuous and transient 822 monitoring profiles in event-based prospective memory. Psychon. Bull. Rev. 22, 492-499 823 (2015).

824 54. Cohen, A.-L., Gordon, A., Jaudas, A., Hefer, C. \& Dreisbach, G. Let it go: the flexible 825 engagement and disengagement of monitoring processes in a non-focal prospective 826 memory task. Psychol. Res. 81, 366-377 (2017). 
827 55. Kuhlmann, B. G. \& Rummel, J. Context-specific prospective-memory processing: Evidence

828 for flexible attention allocation adjustments after intention encoding. Mem. Cognit. 42,

8299 943-949 (2014).

830 56. Hicks, J. L., Marsh, R. L. \& Cook, G. I. Task interference in time-based, event-based, and dual

831 intention prospective memory conditions 录. J. Mem. Lang. 53, 430-444 (2005).

832 57. Momennejad, I. \& Haynes, J.-D. Human anterior prefrontal cortex encodes the 'what' and

833 'when' of future intentions. Neurolmage 61, 139-148 (2012).

834 58. Momennejad, I. \& Haynes, J.-D. Encoding of Prospective Tasks in the Human Prefrontal

835 Cortex under Varying Task Loads. J. Neurosci. 33, 17342-17349 (2013).

836 59. Gilbert, S., J. Decoding the content of delayed intentions. J. Neurosci. 31, 2888-2894 (2011).

837 60. Oksanen, K. M., Waldum, E. R., McDaniel, M. A. \& Braver, T. S. Neural Mechanisms of Time-

838 Based Prospective Memory: Evidence for Transient Monitoring. PLoS ONE 9, e92123 (2014).

839 61. Bowden, V., K., Smith, R. E. \& Loft, S. Eye Movements Provide Insights into the Conscious

$840 \quad$ Use of Context in Prospective Memory. Conscious. Cogn. 52, 68-74 (2017).

841 62. Wagenmakers, E.-J. \& Farrell, S. AIC model selection using Akaike weights. Psychon. Bull.

842 Rev. 11, 192-196 (2004).

843 63. West, R., Carlson, L. \& Cohen, A.-L. Eye movements and prospective memory: What the

844 eyes can tell us about prospective memory. Int. J. Psychophysiol. 64, 269-277 (2007).

845 64. Zuber, S., Kliegel, M. \& Ihle, A. An individual difference perspective on focal versus nonfocal

846 prospective memory. Mem. Cognit. 44, 1192-1203 (2016).

847 65. Grill-Spector, K. \& Weiner, K. S. The functional architecture of the ventral temporal cortex

848 and its role in categorization. Nat. Rev. Neurosci. 15, 536-548 (2014). 
849 66. D’Esposito, M. \& Postle, B. R. The Cognitive Neuroscience of Working Memory. Annu. Rev. $850 \quad$ Psychol. 66, 115-142 (2015).

851 67. Kriegeskorte, N., Goebel, R. \& Bandettini, P. Information-based functional brain mapping. $852 \quad$ Proc. Natl. Acad. Sci. 103, 3863-3868 (2006).

853 68. Oosterhof, N. N., Connolly, A. C. \& Haxby, J. V. CoSMoMVPA: Multi-Modal Multivariate 854 Pattern Analysis of Neuroimaging Data in Matlab/GNU Octave. Front. Neuroinformatics 10, 855 (2016).

856 69. Boywitt, D., C. \& Rummel, J. A diffusion model analysis of task interference effects in 857 prospective memory. Mem. Cognit. 40, 70-82 (2012).

858 70. Heathcote, A., Loft, S. \& Remington, R., W. Slow Down and Remember to Remember! A 859 Delay Theory of Prospective Memory Costs. Psychol. Rev. 122, 376-410 (2015).

860 71. Strickland, L., Heathcote, A., Remington, R. W. \& Loft, S. Accumulating evidence about what 861 prospective memory costs actually reveal. J. Exp. Psychol. Learn. Mem. Cogn. 43, 16168621629 (2017).

863 72. Loft, S., Bowden, V. K., Ball, B. H. \& Brewer, G. A. Fitting an ex-Gaussian function to examine 864 costs in event-based prospective memory: Evidence for a continuous monitoring profile.

$865 \quad$ Acta Psychol. (Amst.) 152, 177-182 (2014).

866 73. Sobel, K. V., Gerrie, M. P., Poole, B. J. \& Kane, M. J. Individual differences in working 867 memory capacity and visual search: The roles of top-down and bottom-up processing. $868 \quad$ Psychon. Bull. Rev. 14, 840-845 (2007). 
869 74. Bisiacchi, P. S., Schiff, S., Ciccola, A. \& Kliegel, M. The role of dual-task and task-switch in 870 prospective memory: Behavioural data and neural correlates. Neuropsychologia 47, 1362$871 \quad 1373$ (2009).

872 75. Li, X., Morgan, P. S., Ashburner, J., Smith, J. \& Rorden, C. The first step for neuroimaging 873 data analysis: DICOM to NIfTI conversion. J. Neurosci. Methods 264, 47-56 (2016).

874 76. Fischl, B. FreeSurfer. Neurolmage 62, 774-781 (2012).

875 77. Jenkinson, M., Beckmann, C. F., Behrens, T. E. J., Woolrich, M. W. \& Smith, S. M. FSL.

$876 \quad$ Neurolmage 62, 782-790 (2012).

877 78. Avants, B. B. et al. A reproducible evaluation of ANTs similarity metric performance in brain

878 image registration. Neurolmage 54, 2033-2044 (2011).

879 79. Horn, S. S., Bayen, U. J. \& Smith, R. E. Adult age differences in interference from a 880 prospective-memory task: a diffusion model analysis. Psychon. Bull. Rev. 20, 1266-1273 $881 \quad$ (2013). 